\section{DIABETES INSIPIDUS COMPLICATED BY INTERMITTENT} GLYCOSURIA.

By GEOFFREY EVANS, M.B. CANTAB., M.R.C.P. LOND.,

PHYSICIAN TO OUT-PATIENTS, METROPOLITAN HOSPITAL: CHIEF ASSISTANT TO A MEDICAL UNIT, ST, BARTHOLOMEW'S AND

R. L. MACKENZIE WALLIS, M.A., M.D. Cantab., CHEMICAI PATHOLOGIST TO ST. BARTHOLOMEW'S HOSPITAI IECTURER IN CHEMICAL PATHOLOGY, ST. BARTHOLOMEW'S
HOSPITAL MEDICAL SCHOOL.

THE earliest record of the occasional presence of traces of sugar in the urine of diabetes insipidus is a reference to two cases by Sir William Roberts' in 1876. Naunyn ${ }^{2}$ in his monograph on diabetes mellitus refers to a single case in his own experience, but no details are given. Von Noorden ${ }^{3}$ mentions the fact of transitory glycosuria in diabetes insipidus, though he does not discuss the condition nor describe a case. The only complete record of a case of intermittent glycosuria that we have found is one reported by Mackenzie Wallis and Roper. ${ }^{4}$ The main feature of this case was the association of glycosuria with "acidosis" and creatinuria. The presence of polyuria was noted, but its bearing on the case was not discussed. In the light of the two cases here recorded it is probable that the case was of similar nature.

\section{Clinical Record.}

CASE 1.-A. Z., age 32, of Jewish nationality, a market porter, was admitted to St. Bartholomew's Hospital under the care of Dr. J. H. Drysdale, on 22/1/20, complaining of loss of energy and strength. The man ascribed his symptoms to the strain of army service, and stated that he had good health until the spring of 1918, when he first complained of a dull pain in the small of the back. He went sick in May, 1918, on account of this pain, and about this date he first complained of thirst, weakness and drowsiness. He described his weakness as a tired feeling, a "droopy feeling," as though he had no life in him. This feeling was liable to occasional aggravation, for which he used the word "drowsiness." At the same time he suffered from a "tight feeling" over both eyes (deep supra-orbital), which sometimes amounted to a headache. $\mathrm{He}$ was admitted with these symptoms to the Mile-End Military Hospital, where sugar was found in the urine, and the case was diagnosed as diabetes mellitus. He was told not to eat potatoes, and he was invalided from the army on his discharge from hospital in July, 1918. After his discharge from the army he returned to his work as a market porter, but after six weeks' work he was unable to continue on account of pain in the back and weakness. The symptoms as outlined above have persisted and varied little since their onset. There is no history of past illness, and V.D. is denied. It appears from his statements that he has had polyuria for the past ten years.

Family history.-Father and mother alive and well. Wife healthy, and has had no miscarriages; he has two childrenone, aged 8, is delicate and has had pneumonia twice; the other, aged 4, has "always seemed to pass a lot of water," and in infancy suffered from enuresis. She is otherwise bright and active, and a single morning specimen of urine brought for examination was normal.

Condition on admission. - The salient facts observed on admission were noted from time to time unchanged from the date that he first came under observation in May, 1919. General appearance: small build, good physique, no skeletal deformity. Skin and hair natural. The upper lids drooped somewhat and the eyes were a little prominent; the sclerotics were always somewhat congested, so that the facies suggested a man who has been drinking the previous night. The tonsils were hypertrophied, there was marked pyorrhcea alveolaris. Eyes: pupils equal, normal reaction to light and accommodation; optic discs and visual fields normal. In the neuro-muscular system there were no abnormalities, except that neither the knee-jerks nor the ankle-jerks could be elicited; other reflexes were normal but weak. Cardiovascular system : normal. Blood pressure 113/68. Kidneys: the following figures give the fluid intake and output in ounces on successive days under observation in hospital-fluids/urine, 160/136, 192/146, 90/80, 172/-, 140/80, $140 / 148,147 / 110,110 / 92,101 / 104,84 / 60,62 / 60,86 / 96,87 /-$. The following figures give the specific gravity of 24-hour specimens; 1008, 1010, 1010, 1010, 1015, 1012, while it was on two frequency of micturition, and the above figures show the amount of polyuria. During the six weeks that the patient was under the observation of Dr. W. Langdon Brown in 1919 sugar was only found in the urine in very small amounts on two occasions. In general the urine was clear, pale amber in colour, with a nebula deposit, never contain. ing albumin or diacetic acid.

The blood Wassermann reaction was negative. The diastase in the urine was 22 units. $X$ ray examination of the skull on 13/9/19 by Mr. Martin Berry showed a slightly expanded sella turcica, and the posterior clinoid processes appeared thinned; a faint shadow was visible in the region of the pituitary body. From the skiagram it did not appear that the condition was necessarily beyond the limits of normal variation. A later skiagram taken by Mr. H. George in February, 1920, was reported on as follows: "The pituitary fossa is a little on the large side, its shadow clinoid processes are somewhat thinned and the sella turcica somewhat enlarged."

Other points noted were as follows: the hands were small, the fingers markedly square-ended, the feet and toes were of normal shape, as were the ears and nose. Constipation was the rule; sleep was undisturbed and appetite good. Loewi's reaction was negative.

CASE 2.-P. M., age 35, war-pensioner and previously a gasworks stoker, was admitted to St. Bartholomew's Hospital under the care of Dr. Drysdale, 28/2/20, having been sent to Dr. Mackenzie Wallis by Dr.A. S. Woodwark for a blood-sugar analysis. His chief complaint was of headache and pains in the small of the back. On account of the clinical resemblance of the case to the foregoing patient, who was at that time in the hospital, he was admitted for examination. When in France, working in a Labour Corps behind the line, and shortly after rejoining his unit from sick leave in England, he complained of the onset at one time in October, 1918, of thirst, polyuria, and headache. The headache was a pain across the right eye just above the eyebrow. At about the same time he first complained of pain in the small of the back. He was kept under observation and was told that sugar had been found in his urine. He was ordered a restricted carbohydrate diet consisting of meat, dry toast and milk, without jam, sugar, or potatoes. Fourteen days later he was free from headache and thirst and felt much better; he accordingly rejoined his unit. Shortly afterwards he complained of pain in his stomach; this was diagnosed as dental dyspepsia. From this date on he passed from one hospital to another, only once returning to his unit for one week, being occupied, apparently without any particular symptoms of disease, until his demobilisation in November, 1919, in dental treatment. Nine days after his return home in November, 1919, he complained again of the onset of headache, thirst, and pain in the back. These symptoms persisted without variation until his admission to the hospital. He has had polyuria as long as he remembers, and has had difficulty in holding his water; he has to pass it once or twice during the night. Except for occasional quinzies no past illnesses. Venereal disease was denied. In May, 1915, he was rejected for the army on account of being roundshouldered and deformity of the feet (hammer toe). In July, 1918, having been recruited for a Labour Corps in the meantime, he was invalided home from France with inflammation of connective tissues, legs and feet. It was after rejoining his unit from his illness that his pituitary symptoms first developed.

Condition on admission.-General appearance : considerable growth of coarse dark hair in all the normal regions It was a marked feature of his appearance, though not beyond the normal variation. The skull was dolichocephalic and the lower jaw prominent. There was marked dorsal kyphosis and some degree of lumbar lordosis; the kyphosis was attributed by the patient to carrying a heavy weight on the back soon after leaving school. No other skeletal deformity was present. He was a man of low intelligence, but in other respects cerebration was normal. In addition to the symptoms already noted he complained of a feeling of tiredness; he explained this symptom by standing at attention and saying that he could not stand thus, but only like this, and then he let his shoulders flop forward and stood with knees a little flexed. The tongue was clean and moist; the tonsils hypertrophied; all teeth except three were missing, and their place taken by false dentures. The cardiovascular system was normal. The heart gave evidence of slight hypertrophy, and there was slight thickening of the radial artery. These signs were not more than might be expected in a gasworks stoker 35 years old. The blood pressure was $110 / 65$. The neuromuscular system was normal. The optic discs were normal. There was no refractive error; the visual fields were normal; all reflexes were brisk and natural. There was no evidence of kidney disease. The urine on boiling gave a faintest cloud; there was, however, no precipitate with salicyl-sulphonic acid, and this was decided to establish the fact that no albumin was present. Many specimens were examined for casts; occasional hyaline casts were found in centrifuged 
deposits both from fresh and 24-hour specimens, but no abnormal deposit was found. From this aspect the urine was therefore normal. During the period of the patient's observation in hospital a trace of sugar was found in the urine in four specimens. This sugar was identified as glucose. The following figures give the range of variation of fluid output and intake for 24-hour periods: Intake Urine $=-164,-/ 34$ plus, 84 plus $/ 108,81 / 72,78 / 90,81 / 84,95 / 112$, $72 / 120,88 / 106,102 / 106,91 / 104,82 / 112,97 / 100,91 / 98,99 / 90$; these figures do not correspond accurately, as the patient was in fome respects difficult to control. The following figures give the range of variation in specific gravity of 24-hour specimens : 1013, 1005, 1010, 1010, 1011, 1007, 1010, 1010. The urine was pale, slightly hazy, though perfectly clear immediately it was passed. The faint haze on boiling not due to albumin led to a suspicion of cystitis, although there was no other evidence of this complaint. A catheter specimen of the urine was examined bacteriologically, but both aerobic and anaerobic cultures were negative. Diacetic acid was never found in the urine. As regards other tests, the blood Wassermann reaction was negative; the diastase in the urine was $22 \cdot 2$ unitg (normal). Loewi's reaction was positive on March 5th, though negative at a later date (15/3/20).

Without other treatment than rest in bed the patient suddenly recovered of his symptoms on March 6th. He said on the following day that he felt quite well, that he had lost his headache and pain in his back, and that his energy had returned. There were no outward signs of this change in his condition. The polyuria continued as before, and though he seemed to drink as much as previously he said that he had lost the thirst of which he had previously com plained. While not attaching too great importance to signs whose significance is still an uncertain matter, it is noted that the Loewi's reaction was positive while he felt ill and negative when he felt that he had recovered. A difference in the response to sugar was also noted.

\section{Chemical Pathology.}

Technique.-Specimens of urine were repeatedly tested for sugar by chemical and polarimetric methods. A negative reaction with Benedict's solution was regarded as indicating the absence of sugar in patho logical quantity. Blood sugar determinations were made by the writer's method," and the diastatic activity of the urine by a modification of the original Wohlgemuth method.

CASE 1. $-15 / 9 / 19$. Blood sugar $=0.1$ per cent. (normal). January-February, 1920, sugar tolerance tests :-28/1/20 6. 100 . 0.595 per cent. sugar, total $1.13 \mathrm{~g}$.; $7-8$ A.M., vol. $160 \mathrm{c.cm}$. 0.356 per cent. sugar, total $0.569 \mathrm{~g} . ; 8-9$ A.M., vol. $165 \mathrm{c.cm}$., 0.00 per cent. sugar, total -;9-6 A.M., vol. $1770 \mathrm{c.cm}$. 0.356 per cent. sugar, total $6.3 \mathrm{~g}$. Report: The patient passed 0.1 per cent. sugar in the urine before the test, and this amount was increased after taking $100 \mathrm{~g}$. glucose. The tolerance appears, therefore, to be less than $100 \mathrm{~g}$. and more than 50 g. $9 / 2 / 20: 6$ A.M., 75 g. glucose taken. Urine: 6-7 A.M., vol. $200 \mathrm{c.cm} ., 0.2 \mathrm{~g}$. per cent. sugar, total $0.4 \mathrm{~g}$ Urine examined in hourly specimens afterwards contained no sugar. Report: These observations show that the sugar tolerance of this patient lies above 50 and below $100 \mathrm{~g}$. 5/2/20: 10.50 A.M., blood sugar 0.09 per cent., urine no sugar 11 A.M., 25 g. glucose taken ; 11.15 A.M., blood sugar $0 \cdot 122$ per cent.; 11.30 A.M., blood sugar, 0.137 per cent.; 12 noon, blood sugar 0.09 per cent. : 12.30 P.M., blood sugar, 0.09 per cent. Trine, 11 A.M. -12 noon, contained 1 per cent. sugar. Total vol. 245 c.cm. Total sugar, $2.45 \mathrm{~g}$. Report: Tolerance on this day was lowered. The blood sugar showed a practically normal change following $25 \mathrm{~g}$. glucose, but the threshold value was lowered.

CASE 2.-2/3/20: Before test, blood sugar 0.11 per cent.; urine, no sugar. 11 AM., $50 \mathrm{~g}$. gluzose taken by mouth. 11.15 A.M., blood sugar 0.207 per cent. 11.30 A.M., blood sugar 0.307 per cent. 12 noon, blood sugar 0.226 per cent. Urine, 11 A.M. -12 noon contained 0.7 per cent. sugar; total volume $310 \mathrm{c.cm}$; t total sugar $2 \cdot 2$ g. Report: The blood sugar showed a definite response to the ingestion of $50 \mathrm{~g}$. glucose, the marked hyperglycæmia being maintained for over one hour. It is evident from the figures that the leak point for sugar is high but the tolerance low.

After the patient had made a clinical recovery another tolerance test was performed with the following result: 15/3/20: Before test, blood sugar $0 \cdot 1$ per cent.; urine, no sugar. 11 A.M., $50 \mathrm{~g}$. glucose taken by mouth. 11.15 A.M., blood sugar 0.113 per cent. 11.30 A.M., blood sugar, 0.140 per cent. 12 noon, blood sugar, 0.122 per cent. 12.30 P.M., blood sugar, 0.110 per cent. 1 P.M., blood sugar 0.09 per cent. Urine, 11 A.M. -12 noon, contained no sugar; urine, 12 noon1 P.M., contained no sugar. Report: The lowered glucose tolerance observed 13 days previously had disappeared, corresponding with the improvement in the condition of the patient. The response to the sugar ingestion was normal, and there was no glycosuria.
The nature of the reducing substance in the urine in both patients was established as being glucose by the fact of its being dextro-rotatory, and by the preparation of glucosazone crystals.

Discussion.

The most constant and important signs of disease shown by these two patients were polyuria and the secretion of urine of low specific gravity. Chemical examination of the urine, microscopical examination of its deposit, and clinical examination of the cardiovascular system ruled out renal disease as a cause of the abnormal condition of the urine. Nor can it be ascribed to polydipsia or to gross disease of any part of the nervous system, in so far as such disease can be excluded by clinical examination. It is considered that, in the absence of demonstrable functional or organic disease of the central nervous system and kidneys, the diagnosis of diabetes insipidus is justified in the presence of these two signs; there was evidence in both cases that the complaint was of long standing. The cases are examples of the complaint in its slightest form, since their excretion of urine was not often more than 4 litres in the 24 hours and was some days as little as 2 litres, while the specific gravity of the wrine was generally about 1010 in a 24-hour specimen, and sometimes normal, in a single specimen.

The association of diabetes insipidus with pituitary disease has long been recognised. More recently G. L. Kennaway and J. C. Mottram ${ }^{6}$ have reported two cases of diabetes insipidus in which there was evidence of pituitary involvement, and in this communication they give an account of present knowledge of the relation of pituitary disease to diabetes insipidus. These observers come to the conclusion that " the existing evidence of morbid anatomy seems on the whole to be distinctly in favour of an association between lesions of the glands and the disease in question." Though disease of the pituitary gland may occur without polyuria there do not appear to be any cases on record in which diabetes insipidus occurred and in which the pituitary was proved post-mortem to be normal, or where it was normal the stalk free from any pressure. Physiological evidence supports pathological evidence on this point. Thus Swale Vincent ${ }^{7}$ states that "so far the only symptom of acromegaly which appears to have a direct relation to the function of the pituitary, as revealed by physiological experiment, is polyuria.", From the clinical aspect we know polyuria as a frequent symptom in both acromegaly and dystrophia adiposisgenitalis. Neither patient showed any very obvious sign of pituitary disease, but in spite of this, on the strength of the general evidence outlined above, it is justifiable to diagnose both cases as pituitary dystrophies and to inquire particularly for the presence of other signs in support of this diagnosis. Case 1 had an enlarged sella turcica, an enlargement which of itself is not sufficient perhaps to determine a pathological condition, but of importance in relation to the facies (the slight exophthalmos and congestion of the sclerotics) and the general consideration of the case. Case 2 varied from the normal in the direction of acromegaly in the following skeletal features: the lower jaw was underhung, the skull dolichocephalic, and there was dorsal kyphosis; hirsuties was marked.

Both patients made chief complaint of headache and definite complaint of lassitude. This lack of energy is a common complaint in pituitary disease, varying from a feeling of inertia and drowsiness to remarkable periods of somnolence. The headache in both cases was frontal; in Case 1 it was bilateral and in Case 2 limited to the right supra-orbital region. Headache is a constant symptom in pituitary disease. In the cases of pituitary disease described by K. Falta ${ }^{8}$ and Harvey Cushing ${ }^{9}$ headache is rarely absent. Cushing regards a bitemporal headache as the more characteristic of pituitary disease. At the same time, many of the patients whose histories he gives complained of a severe frontal headache. In our experience frontal headache situated deep in the supra-orbital region, and described by the patient as a tight feeling or gnawing pain, is not uncommon in pituitary disease, and Pardee ${ }^{10}$ has lately drawn atten. tion to the frontal distribution and intractability of the headache in pituitary disorders. 
There remains for consideration the intermittent glycosuria exhibited by both patients. This glycosuria is in no sense an early phase of diabetes mellitus on account of its slight degree, its intermittent nature, its non-progressive course, and its independence of diet. In neither case did a large carbohydrate meal lead to the appearance of sugar in the urine. The intermittent nature of the glycosuria distinguishes the cases from diabetes innocens, and it is of interest that one of the cases recorded by $\mathrm{H}$. Salomon ${ }^{11}$ in his original communication on diabetes innocens complained of polyuria, and did not therefore perhaps belong to his group. Apart from the case described by Mackenzie Wallis and Roper, the only group of cases to which our cases bear any resemblance are the intermittent glycosurias observed in acromegaly. Sir Archibald Garrod ${ }^{12}$ has written that the glycosuria in some cases of acromegaly is intermittent "although the patient's diet was by no means poor in carbohydrate." In the same context he notes that "in the newer literature of pituitary tumours, since 1886 , Borchard found no record of glycosuria apart from acromegalic symptoms." The chemical examinations of the blood sugar establish the importance of these small traces of glucose occasionally found in the urine in these patients. These researches indicate a lower sugar tolerance in both cases and a lowered leak point in one case, and they further lead to the prospect of new light being thrown on this aspect of sugar metabolism.

In the present state of our knowledge the positive Loewi's reaction observed in Case 2 is to be regarded as an indication of a polyglandular dystrophy, and not necessarily as a sign of pancreatic disease. The association of pancreatic disorder with acromegaly is referred to by several writers ( $\nabla$. Falta), but in our patients there was no evidence of pancreatic disease other than the glycosuria; the diastase reaction was normal in both.

\section{Summary.}

The two patients here described appear to be examples of a pituitary dystrophy comparable to those clinical cases of thyroid dystrophy in which there is no marked change in the size of the gland and no distinct clinical picture of either hypo- or hyperfunction. From their past history neither case seems to be progressive, though Case 2 is liable to periodic variations. These patients are not therefore regarded as early cases of acromegaly or other recognised pituitary syndrome, but rather as persons in whom the pituitary is functioning irregularly and perhaps at times excessively. We believe that cases of the type here described have not been previously recognised. The two patients are alike in their salient features, and they do not fit into any of the subdivisions into which Cushing divides his five main groups of dyspituitarism. The interest of the cases does not lie in the possibility of their representing a group of dyspituitarism that has hitherto escaped recognition, but depends on the fact that such cases may lead to the recognition of clinical phases of pituitary activity that bridge the gap between physiological and pathological states. When this borderland has been explored the earliest stages of progressive pituitary disorders will be more easily recognised. Cushing remarks that "it would be precocious to make a diagnosis of ' acromegaly' before enlargement of the acral parts of the body occurs. Still it would be possible." Though our two cases are not regarded as early cases of acromegaly, they do bring nearer the possibility that Cushing foresees.

We wish to thank Dr. Langdon Brown for permission to make use of his observations on Case 1 , when the patient was under his care in the autumn of 1919 , Mr. Foster Moore for his examination of the fundus oculi of both patients; and Mr. George who gave us the advantage of his special technique in the measurement of the pituitary fossæ by X ray photography. Dr. E. I. Lloyd, house physician, was responsible for the care of the patients and for carrying through the routine of their examination. We are indebted to Dr. Drysdale for permission to publish these records of his cases.

(Continued at foot of next column.)

\section{THE CULTURAL DIAGNOSIS OF ENTERICA IN INOCULATED INDIVIDUALS.}

\section{BY J. C. G. LEDINGHAM, C.M.G., M.A., D.SC.} M.B., CH.B. ABERD.

CHIEF BACTERIOLOGIST, LISTER INSTITOTE, LONDOR.

THERE appears to be an impression that preventive inoculation against enterica diminishes the chance of recovering the causative organism from the blood. Thus, in a recent paper by R. P. Garrow, ${ }^{2}$ entitled "The Myth of 'Atypical' Enteric Fever," with the argument of which I am in sympathy, in substance at least if not in detail, the following statement occurs:-

"The difficulty of recovering enteric bacilli from the blood of inoculated soldiers is well known, even in the early stages of illnesses which clinically resemble enteric."

Is this a well-known fact? If so, I am not aware of any published data in its support. In bacteriological circles on the Western front during the late war it would appear that a similarly pessimistic view was held. Thus A. E. Webb-Johnson, ${ }^{2}$ writing of his experiences at a stationary hospital in France, remarks :-

"With regard to inoculation there is another point, which is possibly one of the most important. The specific bacteria are not found in either blood, fæces, or urine with anything like the same frequency nor in such numbers as in the non-inoculated, since the microbes are killed more rapidly and effectually, owing to protective ubstances being in the body ready for the attack."

The fostering of such views gave, I imagine, the impetus to that concentration on agglutination tests which formed such a feature of enteric diagnosis in France. Largely on that basis of diagnosis cases of enterica have been analysed from the standpoint of clinical syndrome, and for some time it has been current teaching that inoculation profoundly modifles the type and severity of the attack in inoculated individuals. With this aspect of the case I am not immediately concerned, but I hold that any comparative analysis of clinical syndrome or of incidence and mortality statistics in inoculated and uninoculated individuals must, to be above all reproach, be founded on a culturally diagnosed series of cases.

Analysis of 373 Cases in American Army.

This has been done in a recently published and most valuable article by the late Major V. C. Vaughan, ${ }^{3}$ of the United States Army, whose conclusions are in agreement with those of Garrow (loc. cit.). This author submits with full bacteriological particulars a clinical analysis of 373 cases of enterica (mainly typhoid) which occurred among American troops in France, all of whom had been inoculated with the triple vaccine. For the purpose of his analysis the following proviso was laid down :-

"No case has been accepted for our statistical study as being undoubted typhoid or paratyphoid fever unless the causative organism has been isolated either from the urine or fæces, or pre ferably from the blood. I recognise that the finding of the typhoid or a paratyphoid bacillus in the freces alone does not establish diagnosis, but have assumed that this finding, together with the presence of characteristic clinical symptoms of the disease and with negative blood cultures, form sufficient evidence on which to base a diagnosis."

A series of 270 cases in which B. typhosus was recovered from the blood or excreta $(180$ by blood culture) is taken for the main clinical study. His conclusions cannot be summarised here, and I need only say that the results of the analysis went to show that vaccination had not altered the essential characters of the disease.

(Continued from previous columm)

References.-1. Roberts, Sir William: Urinary and Renal Diseases, third edition, 1876, p. 217. 2. Naunyn: Der Diabetes Mellitus, 1906 p. 49. 3. Von Noorden: Handbuch d. Pathol. des Stoff wechsels, 1907, ii. p. 881. 4. Mackenzie Wallis and Roper: A Case of Intermitten Grycosuria, St. Bart.'s Hospital Reports, vol. Xlvili. 5. Mackenzi Walls and Gallagher: THE LANCET, Oct. 16th, 1920. 6. Kennawa. and Mottram: Quarterly J1. of Med., 1919, xil., p. 247. 7 . Swal Vincent Int. Secretions and the Ductless Glands, 1912, p. 379 8. Falta : Die Erkrankungen d. Blutarusen, 1913, p. 189. 9. Cushing The Pituitary Body and its Disorders, 1912. 10. Pardee : Areh. Int Med., February, 1919. 11. Salomon : Deutsch. med. Woch., Berlin 1914, xl., 1, 217. 12. Garrod: Lettsomian Lectures, TrE LARCET.
Oct. 16th, 1912. 